\title{
ANÁLISIS COMPARATIVO DE LA DINÁMICA DE DESARROLLO AGRÍCOLA EN SURAMÉRICA EN EL PERÍODO 1980-2010
}

\author{
Holmes Rodríguez Espinosa ${ }^{1}$ \\ Carlos Julián Ramírez Gómez ${ }^{2}$ \\ Luis Fernando Restrepo-Betancur ${ }^{3}$
}

Recibido el 26 de agosto de 2014, aprobado el 22 de abril de 2015 y actualizado el 22 de octubre de 2015

DOI: 10.17151/luaz.2016.42.3

\section{RESUMEN}

El desarrollo agrícola de las naciones ha sido un foco de gran importancia y controversia, en particular por la forma en que se han orientado diversas políticas públicas en Suramérica, así como por los diversos enfoques, objetivos y alcances logrados. Dadas las particularidades específicas de los países y sus territorios rurales, y en vista de los resultados disímiles alcanzados en materia de crecimiento y desarrollo, este estudio pretende realizar un análisis comparativo del desarrollo agrícola de las naciones suramericanas, a partir del abordaje de diversos indicadores que permiten contribuir a interpretar la heterogeneidad en los resultados alcanzados en el sector. Se utilizó análisis multivariado de la varianza (MANOVA) y análisis de componentes principales. Se encontró diferencia altamente significativa entre países $(p<0,0001)$ en lo relacionado con los factores: proporción del área destinada a la agricultura, porcentaje de población rural y empleo generado en el sector rural. Se encontró que existe una brecha en los indicadores del desarrollo de la agricultura entre algunos países de Suramérica, destacándose Argentina y Paraguay como los que presentan un crecimiento significativo en el mayor número de indicadores.

\section{PALABRAS CLAVE}

Desarrollo rural, políticas agrarias, análisis multivariado.

\section{COMPARATIVE ANALYSIS OF AGRICULTURAL DEVELOPMENT DYNAMICS IN SOUTH AMERICA IN THE PERIOD 1980-2010}

\begin{abstract}
The agricultural development of each nation has been a focus of great importance and controversy, particularly because of the way several public policies have been oriented in South America, as well as the different approaches, objectives and outcomes achieved. According to the specific characteristics of countries and their rural territories, and because of the dissimilar results achieved in terms of growth and development, this study aims to provide a comparative analysis of agricultural development of South American nations from the approach to diverse indicators which contribute to interpret the heterogeneity in the results achieved in the sector. Multivariate analysis of variance (MANOVA) and analysis of the main components was performed. Highly significant difference between countries $(p<0.0001)$ in relation to factors such as proportion of land in agricultural use, rural population and rural employment, was found. It was found that there is a gap in the agricultural development indicators between some South American countries, being Argentina and Paraguay those with a significant growth in the largest number of indicators.
\end{abstract}

KEY WORDS: Rural development, agricultural policies, multivariate analysis. 


\section{INTRODUCCIÓN}

Las políticas de liberalización y desregulación de los mercados producto de los procesos de apertura económica implementados en los países suramericanos en la segunda mitad del siglo XX, estaban orientados a lograr una agricultura más eficiente y productiva basada en la especialización productiva a partir de las ventajas comparativas de cada país.

Como resultado de estas políticas, se han presentado importantes cambios en el sector agrícola con comportamientos disímiles en cada país, acordes con las diferencias socioeconómicas y estructurales de cada nación, que en términos generales han traído consigo dificultades para los países suramericanos en términos del aumento de la migración internacional, incremento del sector informal urbano y las desigualdades económicas (Baudasse y Calderón, 2009). Adicionalmente, han conllevado a una consolidación de la agricultura comercial con destino a la exportación que se beneficia de la infraestructura, créditos y fertilizantes, pero que no resuelve los problemas de empleo rural (Soto, 2003).

El desarrollo en regiones rurales depende de múltiples factores de tipo económico, social y político (Lowe et al., 1993). En términos generales, el desarrollo económico puede evidenciarse por el crecimiento del PIB y del PIB per cápita (Armstrong y Taylor, 2000); sin embargo, la noción de crecimiento económico va acompañada de las dimensiones sociocultural y política (Moulaert, Sekia y Boyabe, 1999), en la cual se genera una relación importante entre el Estado como garante de la planificación e implementación de políticas de desarrollo y los beneficiarios como actores garantes de la sostenibilidad del desarrollo local (Terluin, 2003).

En este sentido, de acuerdo con los planteamientos de Porter (2007), para lograr el desarrollo, además de la capacidad de un país de inserción al mercado global y de atracción de la inversión extranjera, se requiere fortalecer las capacidades básicas competitivas entre las cuales se destacan la infraestructura (vías, energía y recurso hídrico), la educación y el tejido institucional. Costa et al. (2013) por su parte destacan como elementos esenciales para el desarrollo: la calidad de vida en el medio rural, la infraestructura y la inversión pública.

Sin embargo, el desarrollo agrícola plantea tres preocupaciones importantes (Cazalis, 1980): 1) los grandes objetivos de la política agrícola, orientados a lograr una agricultura más eficiente basada en la especialización productiva a partir de las ventajas comparativas de cada país; 2) el ámbito de la producción agraria, la productividad y rentabilidad de las unidades, así como el mejoramiento de las condiciones de trabajo y calidad de vida; 3 ) el territorio en el cual se sitúa la producción, teniendo en cuenta aspectos no agrícolas como los activos rurales, infraestructura, uso del suelo, impacto ambiental y manejo ecológico de pequeñas regiones productivas, así como el mantenimiento de la vida social rural, asuntos económicos, políticos, entre otros (Lowe et al., 1993).

Son diversas las políticas para el desarrollo agrícola, que contextualizadas en un nivel regional y sectorial, han procurado tratar de resolver problemas puntuales del productor en la agricultura suramericana, fundamentalmente en asuntos de la producción, acceso a mercados, organización de productores, logística, entre otros. Algunos países han adoptado políticas en un nivel macro, basadas en la provisión de bienes públicos, desarrollo de la infraestructura, apoyo a la investigación, información, instituciones y programas de educación (FAO, 2004), muchas de las cuales en algunos países de la región, han constituido un factor determinante para incrementar el ingreso y lograr la reducción de la pobreza, 
crecimiento económico y desarrollo social (Kabeer y Anh, 2000 citados por Rigg, 2006).

Otras políticas que también se han implementado de manera heterogénea en los países suramericanos son: incentivos a los productores, tenencia de la tierra, gestión del agua en la agricultura, políticas financieras para el desarrollo agrícola, tecnología agrícola, entre otras (FAO, 2004; Norton, 1992). Sin embargo, el papel del Estado en el desarrollo agrícola, ha venido cambiando a través del tiempo, hacia un manejo menos protagónico en las actividades económicas, menos controles sobre los precios y las cantidades de factores y productos.

En este contexto, es importante realizar el análisis de los resultados de las políticas públicas en el desarrollo agrícola, teniendo en cuenta su impacto en la solución de problemáticas estructurales de la población rural como el acceso a la tierra y a fuentes de trabajo dignas (Gorenstein, Napal y Olea, 2007); así como también otros indicadores para evaluar el desarrollo agrícola planteados por autores como Fawaz y Vallejos (2011), entre los cuales están la dinámica poblacional, el empleo rural, la disponibilidad de servicios como el agua, y la estrategia productiva.

En ese sentido, el objetivo de este estudio es realizar un análisis comparativo del desarrollo agrícola de las naciones suramericanas, a partir del abordaje de diversos indicadores y empleando el análisis multivariado, método utilizado por otros autores para análisis de política agrícola (Delgadillo y Chalita, 2013), con el fin de contribuir a la interpretación de la heterogeneidad que presentan los países en materia de alcances y resultados para su territorio rural.

\section{MATERIALES Y MÉTODOS}

A partir de la revisión de literatura y la noción de varios autores, la información disponible y los planteamientos de Cazalis (1980) sobre el desarrollo agrícola, en sus categorías de eficiencia en la agricultura, la producción agraria y el rol campesino, y el territorio rural bajo una visión multidimensional, se seleccionaron los siguientes indicadores de desarrollo agrícola como variables para este estudio: porcentaje de la población rural, consumo de fertilizantes, porcentaje de empleos generados por la agricultura, porcentaje de la población rural con acceso a agua, rendimiento de los cereales, la contribución de la agricultura al PIB, porcentaje de tierra dedicada a la agricultura, porcentaje de tierra cultivable, área cultivable permanentemente y tierra cultivable por persona; estas variables tenían distribución normal y binomial (Tabla 1). 
Tabla 1. Naturaleza de las variables seleccionadas para el análisis del desarrollo agrícola en Suramérica

\begin{tabular}{lcccc}
\hline Variable & $\begin{array}{c}\text { Tipo } \\
\text { variable }\end{array}$ & $\begin{array}{c}\text { Tipo de } \\
\text { proyección }\end{array}$ & Codificación & Medida \\
\hline País & Cualitativa & Suplementaria & & Categórica \\
Década & Cualitativa & Suplementaria & & Categórica \\
Población rural & Cuantitativa & Activa & PR & Porcentaje \\
Fertilizantes & Cuantitativa & Activa & CF & kg hectárea \\
Empleos & Cuantitativa & Activa & PE & Porcentaje \\
Población con agua & Cuantitativa & Activa & PA & Porcentaje \\
Rendimiento cereal & Cuantitativa & Activa & RC & kg hectárea \\
PIB agrícola & Cuantitativa & Activa & PIB & Porcentaje \\
Tierra en agricultura & Cuantitativa & Activa & TA & Porcentaje \\
Tierra cultivable & Cuantitativa & Activa & TC & Porcentaje \\
Area permanente & Cuantitativa & Activa & AR & Porcentaje \\
Tierra por persona & Cuantitativa & Activa & TP & Porcentaje \\
\hline
\end{tabular}

Fuente: los autores, 2014

Para llevar a cabo el proceso investigativo se diseñó una base de datos teniendo en cuenta la información reportada por el Banco Mundial, por ser la fuente que brindaba mayor información de acuerdo con los objetivos del estudio, y por tratarse de una entidad de reconocida trayectoria en la recopilación y difusión de información de carácter internacional. Se realizó un control de calidad sobre la digitación de la información con el objetivo de validar los resultados generados del proceso estadístico.

Para el análisis estadístico de los datos se utilizó el paquete estadístico SAS versión 9.0. Se empleó análisis multivariado de la varianza (MANOVA) con contraste canónico ortogonal, estableciendo la dimensionalidad de la comparación multidimensional, por medio del criterio de máxima verosimilitud observando el mayor valor propio significativo, se aplicó adicionalmente análisis de componentes principales método $\mathrm{R}$ con variable suplementaria de índole cualitativo (país), anotando que las variables activas eran de naturaleza cuantitativa asociadas a distribuciones probabilísticas de tipo normal. El análisis se complementó por medio de estadística descriptiva de tipo unidimensional.

\section{RESULTADOS}

En la Tabla 2 se pueden observar las comparaciones por década entre países de Suramérica, adicionalmente se describe la tendencia de cada país a través del tiempo, expresado en períodos de 10 años. En el caso de la tierra propicia para labores agrícolas se aprecia a Paraguay con un notable incremento pasando de $38,5 \%$ en la década de los ochenta a $50,9 \%$ para la primera década del nuevo siglo. Uruguay se diferencia significativamente $(p<0,05)$ de los demás países a través de los periodos evaluados con un porcentaje que oscila entre el $84 \%$ y el 
85\%. En cuanto al porcentaje de tierra cultivable, Argentina se diferencia de los demás países de manera significativa $(p<0,05)$.

Tabla 2. Análisis comparativo entre países por década de la evolución en la disponibilidad de tierras

\begin{tabular}{lcccccccccc}
\hline Variable & \multicolumn{3}{c}{$\begin{array}{c}\text { Tierra dedicada a la } \\
\text { agricultura }\end{array}$} & \multicolumn{3}{c}{ Tierra cultivable } & \multicolumn{3}{c}{$\begin{array}{c}\text { Tierra en cultivos } \\
\text { permanentes }\end{array}$} \\
\hline Década & 80 & 90 & 2000 & 80 & 90 & 2000 & 80 & 90 & 2000 \\
\hline País & \multicolumn{3}{c}{$\%$} & & \multicolumn{3}{c}{$\%$} & & & $\%$ \\
\hline Argentina & $46,8 \mathrm{~b}^{*}$ & $46,7 \mathrm{~b}$ & $49,9 \mathrm{~b}$ & $9,7 \mathrm{a}$ & $9,8 \mathrm{a}$ & $11,8 \mathrm{a}$ & $0,4 \mathrm{~d}$ & $0,4 \mathrm{~d}$ & $0,4 \mathrm{e}$ \\
Bolivia & $34,0 \mathrm{~d}$ & $33,4 \mathrm{~d}$ & $34,0 \mathrm{~d}$ & $1,8 \mathrm{~g}$ & $2,2 \mathrm{~g}$ & $3,2 \mathrm{~d}$ & $0,1 \mathrm{f}$ & $0,2 \mathrm{f}$ & $0,2 \mathrm{f}$ \\
Brasil & $27,9 \mathrm{e}$ & $29,6 \mathrm{e}$ & $31,6 \mathrm{e}$ & $5,6 \mathrm{c}$ & $6,4 \mathrm{c}$ & $7,7 \mathrm{~b}$ & $0,8 \mathrm{c}$ & $0,9 \mathrm{c}$ & $0,9 \mathrm{c}$ \\
Chile & $22,1 \mathrm{f}$ & $20,7 \mathrm{~g}$ & $21,0 \mathrm{~g}$ & $4,5 \mathrm{~d}$ & $3,0 \mathrm{e}$ & $1,9 \mathrm{e}$ & $0,3 \mathrm{e}$ & $0,4 \mathrm{~d}$ & $0,6 \mathrm{~d}$ \\
Colombia & $40,8 \mathrm{c}$ & $40,5 \mathrm{c}$ & $38,2 \mathrm{c}$ & $3,3 \mathrm{e}$ & $2,5 \mathrm{f}$ & $1,8 \mathrm{e}$ & $1,3 \mathrm{~b}$ & $1,6 \mathrm{~b}$ & $1,4 \mathrm{~b}$ \\
Ecuador & $26,2 \mathrm{e}$ & $29,5 \mathrm{e}$ & $30,3 \mathrm{e}$ & $5,7 \mathrm{c}$ & $5,9 \mathrm{~d}$ & $5,2 \mathrm{c}$ & $4,0 \mathrm{a}$ & $5,1 \mathrm{a}$ & $5,1 \mathrm{a}$ \\
Paraguay & $38,5 \mathrm{c}$ & $45,1 \mathrm{~b}$ & $50,9 \mathrm{~b}$ & $4,5 \mathrm{~d}$ & $6,2 \mathrm{c}$ & $8,7 \mathrm{~b}$ & $0,3 \mathrm{e}$ & $0,2 \mathrm{f}$ & $0,2 \mathrm{f}$ \\
Perú & $14,7 \mathrm{~g}$ & $16,7 \mathrm{~h}$ & $16,6 \mathrm{~h}$ & $2,6 \mathrm{f}$ & $2,8 \mathrm{e}$ & $2,8 \mathrm{~d}$ & $0,3 \mathrm{e}$ & $0,4 \mathrm{~d}$ & $0,5 \mathrm{~d}$ \\
Uruguay & $85,2 \mathrm{a}$ & $84,9 \mathrm{a}$ & $84,3 \mathrm{a}$ & $7,4 \mathrm{~b}$ & $7,4 \mathrm{~b}$ & $8,2 \mathrm{~b}$ & $0,3 \mathrm{e}$ & $0,3 \mathrm{e}$ & $0,2 \mathrm{f}$ \\
Venezuela & $24,4 \mathrm{e}$ & $24,5 \mathrm{f}$ & $24,1 \mathrm{f}$ & $3,3 \mathrm{e}$ & $2,9 \mathrm{e}$ & $2,9 \mathrm{~d}$ & $0,9 \mathrm{c}$ & $0,9 \mathrm{c}$ & $0,8 \mathrm{c}$ \\
\hline
\end{tabular}

* Comparaciones por columna, letras distintas indican diferencia significativa $(p<0,05)$.

Fuente: los autores, 2014

Al evaluar la población rural, Paraguay presenta diferencia estadística con respecto a los demás países $(p<0,05)$ tal como se aprecia en la Tabla 3. Bolivia por su parte, es el país de Suramérica que más empleo de tipo agrícola genera mientras que Argentina es el de menor porcentaje. La contribución de la agricultura al PIB está disminuyendo en todos los países de Suramérica, con excepción principalmente de Paraguay. Los países con mayor participación de la agricultura en el PIB son Paraguay y Bolivia. 
Tabla 3. Análisis comparativo entre países por década de la evolución de la población rural, el empleo agrícola y la contribución del sector agrícola al PIB

\begin{tabular}{lccccccccc}
\hline Variable & \multicolumn{3}{c}{ Población rural } & \multicolumn{3}{c}{ Empleo agrícola } & \multicolumn{3}{c}{ PIB agrícola } \\
\hline Década & 80 & 90 & 2000 & 80 & 90 & 2000 & 80 & 90 & 2000 \\
\hline País & \multicolumn{3}{c}{$\%$} & & \multicolumn{5}{c}{$\%$} \\
\hline Argentina & $11,7 \mathrm{f}$ & $8,7 \mathrm{f}$ & $8,7 \mathrm{f}$ & $1 \mathrm{e}$ & $1 \mathrm{f}$ & $1,0 \mathrm{f}$ & $8,2 \mathrm{~d}$ & $6,1 \mathrm{~d}$ & $9,5 \mathrm{c}$ \\
Bolivia & $42,1 \mathrm{~b}$ & $35,8 \mathrm{~b}$ & $35,8 \mathrm{~b}$ & $36,5 \mathrm{a}$ & $36,5 \mathrm{a}$ & $36,5 \mathrm{a}$ & $19,1 \mathrm{a}$ & $16,3 \mathrm{a}$ & $14 \mathrm{~b}$ \\
Brasil & $23,3 \mathrm{~d}$ & $17,1 \mathrm{~d}$ & $17,1 \mathrm{~d}$ & $19,3 \mathrm{c}$ & $19,3 \mathrm{c}$ & $19,3 \mathrm{c}$ & $10,5 \mathrm{c}$ & $6,1 \mathrm{~d}$ & $6,1 \mathrm{e}$ \\
Chile & $15,1 \mathrm{e}$ & $12,4 \mathrm{e}$ & $12,4 \mathrm{e}$ & $12,8 \mathrm{~d}$ & $12,8 \mathrm{~d}$ & $12,8 \mathrm{~d}$ & $7,6 \mathrm{~d}$ & $7,9 \mathrm{c}$ & $4,4 \mathrm{f}$ \\
Colombia & $30,1 \mathrm{c}$ & $26,3 \mathrm{c}$ & $26,3 \mathrm{c}$ & $20,2 \mathrm{c}$ & $20,2 \mathrm{c}$ & $20,2 \mathrm{c}$ & $18,4 \mathrm{a}$ & $15,1 \mathrm{~b}$ & $8,1 \mathrm{~d}$ \\
Ecuador & $42,4 \mathrm{~b}$ & $36,2 \mathrm{~b}$ & $36,2 \mathrm{~b}$ & $30,2 \mathrm{~b}$ & $30,2 \mathrm{~b}$ & $30,2 \mathrm{~b}$ & & $17,4 \mathrm{a}$ & $11,1 \mathrm{c}$ \\
Paraguay & $48,1 \mathrm{a}$ & $41,6 \mathrm{a}$ & $41,6 \mathrm{a}$ & $31 \mathrm{~b}$ & $31 \mathrm{~b}$ & $31,0 \mathrm{~b}$ & & $17,5 \mathrm{a}$ & $21,3 \mathrm{a}$ \\
Perú & $29,5 \mathrm{c}$ & $25,5 \mathrm{c}$ & $25,5 \mathrm{c}$ & $30,9 \mathrm{~b}$ & $30,9 \mathrm{~b}$ & $30,9 \mathrm{~b}$ & $13,1 \mathrm{~b}$ & $8,1 \mathrm{c}$ & $7,6 \mathrm{~d}$ \\
Uruguay & $10,1 \mathrm{f}$ & $8,1 \mathrm{f}$ & $8,0 \mathrm{f}$ & $7,5 \mathrm{e}$ & $7,5 \mathrm{e}$ & $7,5 \mathrm{e}$ & $13,1 \mathrm{~b}$ & $8,1 \mathrm{c}$ & $10,3 \mathrm{c}$ \\
Venezuela & $13,1 \mathrm{e}$ & $8,2 \mathrm{f}$ & $8,2 \mathrm{f}$ & $9,5 \mathrm{e}$ & $9,5 \mathrm{e}$ & $9,5 \mathrm{e}$ & $6,0 \mathrm{e}$ & $4,9 \mathrm{~d}$ & $4,5 \mathrm{f}$ \\
\hline
\end{tabular}

* Comparaciones por columna, letras distintas indican diferencia significativa $(p<0,05)$.

Fuente: los autores, 2014

El suministro de agua destinada al sector rural ha ido incrementándose a través de los distintos periodos evaluados, destacándose Uruguay que ha alcanzado una cobertura del 91,4\% para la última década analizada, seguido de Argentina. Perú, Paraguay y Bolivia son los países más rezagados en este aspecto. En cuanto al rendimiento de cereales, se destaca Chile presentando diferencia estadística significativa con respecto a los demás países de Suramérica $(p<0,05)$. Chile pasó de $2831,2 \mathrm{~kg}$ hectárea en la década de los ochenta a 5563,9 kg hectárea en la primera década del 2000 (Tabla 4). 
Tabla 4. Análisis comparativo entre países por década de la población con suministro de agua y el rendimiento de los cereales

\begin{tabular}{lccccc}
\hline Variable & \multicolumn{2}{c}{ Suministro de Agua } & \multicolumn{3}{c}{ Rendimiento Cereales } \\
\hline Década & 90 & 2000 & 80 & 90 & 2000 \\
\hline País & \multicolumn{3}{c}{$\%$} & & \multicolumn{4}{c}{$\mathrm{kg} / \mathrm{ha}$} \\
\hline Argentina & $74,5 \mathrm{~b}$ & $87,8 \mathrm{a}$ & $2371,6 \mathrm{c}$ & $2952,4 \mathrm{~b}$ & $3844,6 \mathrm{~b}$ \\
Bolivia & $47,6 \mathrm{~d}$ & $63,1 \mathrm{~d}$ & $1264,4 \mathrm{e}$ & $1450,9 \mathrm{f}$ & $1902,7 \mathrm{f}$ \\
Brasil & $71,4 \mathrm{~b}$ & $79,8 \mathrm{~b}$ & $1725,8 \mathrm{~d}$ & $2329,7 \mathrm{~d}$ & $3293,1 \mathrm{~d}$ \\
Chile & $56,0 \mathrm{c}$ & $77,8 \mathrm{~b}$ & $2831,2 \mathrm{a}$ & $4293,9 \mathrm{a}$ & $5563,9 \mathrm{a}$ \\
Colombia & $70,3 \mathrm{~b}$ & $71,6 \mathrm{c}$ & $2538,3 \mathrm{a}$ & $2692,9 \mathrm{c}$ & $3792,5 \mathrm{~b}$ \\
Ecuador & $71,6 \mathrm{~b}$ & $78,3 \mathrm{~b}$ & $1699,8 \mathrm{~d}$ & $1894,3 \mathrm{e}$ & $2744,9 \mathrm{e}$ \\
Paraguay & $36,4 \mathrm{~d}$ & $61,0 \mathrm{~d}$ & $1665,2 \mathrm{~d}$ & $2037,9 \mathrm{~d}$ & $2480,1 \mathrm{e}$ \\
Perú & $49,5 \mathrm{~d}$ & $60,0 \mathrm{~d}$ & $2241,9 \mathrm{c}$ & $2688,4 \mathrm{c}$ & $3512,0 \mathrm{c}$ \\
Uruguay & $83,5 \mathrm{a}$ & $91,7 \mathrm{a}$ & $2029,4 \mathrm{c}$ & $2966,9 \mathrm{~b}$ & $4006,2 \mathrm{~b}$ \\
Venezuela & $72,7 \mathrm{~b}$ & $74,9 \mathrm{c}$ & $2017,5 \mathrm{~d}$ & $2903,3 \mathrm{~b}$ & $3350,0 \mathrm{c}$ \\
\hline
\end{tabular}

* Comparaciones por columna, letras distintas indican diferencia significativa $(p<0,05)$.

Fuente: los autores, 2014.

En la Tabla 5 se aprecia que Colombia, Ecuador y Perú presentan en general los mayores patrones de heterogeneidad para las diferentes variables evaluadas.

Tabla 5. Coeficientes de variación por país para las variables evaluadas

\begin{tabular}{|c|c|c|c|c|c|c|c|c|c|c|}
\hline Pais & $\begin{array}{l}\text { Pobl. } \\
\text { rurgl }\end{array}$ & Fertiliz. & $\begin{array}{l}\text { Empleo } \\
\text { agricols }\end{array}$ & $\begin{array}{l}\text { Access } \\
\text { a sgus }\end{array}$ & $\begin{array}{l}\text { Rendim. } \\
\text { ceresles }\end{array}$ & PIB & $\begin{array}{c}\text { Tierrs } \\
\text { sgricols }\end{array}$ & $\begin{array}{l}\text { Tierrg } \\
\text { cultivo }\end{array}$ & $\begin{array}{c}\text { Ares } \\
\text { permanente }\end{array}$ & $\begin{array}{l}\text { Tierrs por } \\
\text { Persons }\end{array}$ \\
\hline Argentins & 24,7 & 15,0 & 25,7 & 15,2 & 12,5 & 13,6 & 12,5 & 12,3 & 21,9 & 34,2 \\
\hline Bolivis & 20,6 & 28,2 & 13,7 & 24,5 & 24,1 & 16,7 & 18,7 & 11,2 & 25,5 & 12,1 \\
\hline Brasil & 0,0 & 9,1 & 10,4 & 9,1 & 7,9 & 5,3 & 7,4 & 8,9 & 46,8 & 5,4 \\
\hline Chile & 9,6 & 16,4 & 6,5 & 19,0 & 1,1 & 5,3 & 28,8 & 11,2 & 5,4 & 1,8 \\
\hline Colombis & 24,0 & 20,2 & 29,6 & 29,7 & 20,1 & 25,2 & 23,0 & 21,5 & 30,3 & 21,3 \\
\hline Ecusdor & 25,5 & 14,7 & 33,2 & 30,4 & 32,4 & 25,9 & 15,2 & 28,5 & 24,5 & 18,6 \\
\hline Parsgugy & 4,1 & 11,2 & 6,9 & 3,2 & 3,2 & 7,7 & 13,2 & 6,0 & 0,9 & 1,2 \\
\hline Perú & 12,1 & 24,8 & 14,6 & 36,2 & 25,5 & 8.2 & 28,1 & 4,6 & 10,3 & 6,6 \\
\hline Urugusy & 0,0 & 26,9 & 9,0 & 29,6 & 10,5 & 13,4 & 20,7 & 27,7 & 19,8 & 7,9 \\
\hline Venezuela & 7,7 & 8,1 & 3,8 & 49,6 & 40,8 & 29,5 & 6,7 & 12,6 & 17,0 & 25,0 \\
\hline
\end{tabular}

Fuente: los autores, 2014.

El análisis multivariado de la varianza determinó diferencia altamente significativa entre los países de Suramérica $(p<0,001)$, para las variables tierra destinada a la agricultura, empleo generado en el sector rural y población existente en el campo, anotando que la técnica multivariada tiene en cuenta un conjunto de variables de manera simultánea, incorporando los factores antes citados (Tabla 6). 
Tabla 6. Análisis multivariado de la varianza (MANOVA) para las variables tierra, empleo y población

\begin{tabular}{lccc}
\hline Prueba estadística & $\begin{array}{c}\text { Década } 80 \\
\text { Valor } p\end{array}$ & $\begin{array}{c}\text { Década 90 } \\
\text { Valor } p\end{array}$ & $\begin{array}{c}\text { Década 2000 } \\
\text { Valor } p\end{array}$ \\
\hline Wilks' Lambda & $<0,0001$ & $<0,0001$ & $<0,0001$ \\
Pillai's Trace & $<0,0001$ & $<0,0001$ & $<0,0001$ \\
Hotelling-Lawley T & $<0,0001$ & $<0,0001$ & $<0,0001$ \\
Roy's Greatest R & $<0,0001$ & $<0,0001$ & $<0,0001$ \\
\hline
\end{tabular}

Fuente: los autores, 2014.

En la Tabla 7 se observa el análisis canónico, el cual se deriva del análisis multivariado de la varianza, determinado previamente la dimensionalidad del contraste a partir de la significancia de los valores propios adscritos a cada espacio. Permitió definir diferencias entre Chile y Uruguay con respecto a los demás países en lo relacionado al tema eficiencia de recursos existentes (suministro de agua, fertilizantes, PIB, rendimiento de cereales), anotando que Chile es el país con menor PIB pero el de mayor producción promedio en el rendimiento de cereales. En lo relacionado con la población rural y empleo, Paraguay presenta diferencia significativa $(p<0,05)$ con respecto a los demás países, donde se da una de las mayores brechas de empleo entre géneros. En el caso de Bolivia, se concentra la mayor población rural con una alta generación de empleo rural, destacándose una similitud entre géneros para este concepto. Brasil se diferencia de los demás países en lo referente al tema de la tierra (tierra agrícola, tierra cultivo, área permanente, tierra por persona).

Tabla 7. Análisis canónico de los componentes tierra (tierra agrícola, tierra cultivo, área permanente, tierra por persona), población rural y empleo y recursos disponibles (suministro de agua, fertilizantes, PIB, rendimiento de cereales)

\begin{tabular}{lccc}
\hline País & Tema tierra & $\begin{array}{c}\text { Tema población } \\
\text { rural y empleo }\end{array}$ & $\begin{array}{c}\text { Tema recursos } \\
\text { disponibles }\end{array}$ \\
\hline Argentina & D & $\mathrm{f}$ & $\mathrm{c}$ \\
Bolivia & $\mathrm{A}$ & $\mathrm{b}$ & $\mathrm{c}$ \\
Brasil & $\mathrm{E}$ & $\mathrm{d}$ & $\mathrm{c}$ \\
Chile & $\mathrm{D}$ & $\mathrm{e}$ & $\mathrm{a}$ \\
Colombia & $\mathrm{C}$ & $\mathrm{c}$ & $\mathrm{b}$ \\
Ecuador & $\mathrm{B}$ & $\mathrm{b}$ & $\mathrm{b}$ \\
Paraguay & $\mathrm{B}$ & $\mathrm{a}$ & $\mathrm{b}$ \\
Perú & $\mathrm{D}$ & $\mathrm{c}$ & $\mathrm{c}$ \\
Uruguay & $\mathrm{D}$ & $\mathrm{f}$ & $\mathrm{a}$ \\
Venezuela & $\mathrm{C}$ & $\mathrm{e}$ & $\mathrm{d}$ \\
\hline
\end{tabular}

Fuente: los autores, 2014. 
El análisis de componentes principales permitió determinar que los factores, en general, asociados con el desarrollo rural no son los mismos entre países tal como se aprecia en la Tabla 8. En el caso de Argentina el primer componente se relaciona positivamente con las variables: acceso de agua, rendimiento cereal, tierra agrícola, tierra cultivo, tierra disponible por persona, mientras existe una correlación negativa con las variables: población rural, PIB. El segundo factor es explicado por los fertilizantes. En el caso de Chile la mayoría de variables se asocian con el primer factor. Para el caso de Colombia el primer componente se explica de manera positiva mediante la asociación con el factor, de las variables: población rural, empleo agrícola, PIB, tierra cultivo, tierra por persona y negativamente se correlacionan las variables: fertilizantes, acceso de agua, tierra agrícola, tierra cultivo. Lo que prueba el análisis de componentes principales, es que no existe articulación entre las variables relacionadas con el desarrollo rural para la inmensa mayoría de países de Suramérica. Chile es el país que más se acerca en asociar todas las variables en único factor y de ahí su eficiencia.

Tabla 8. Análisis de componentes principales

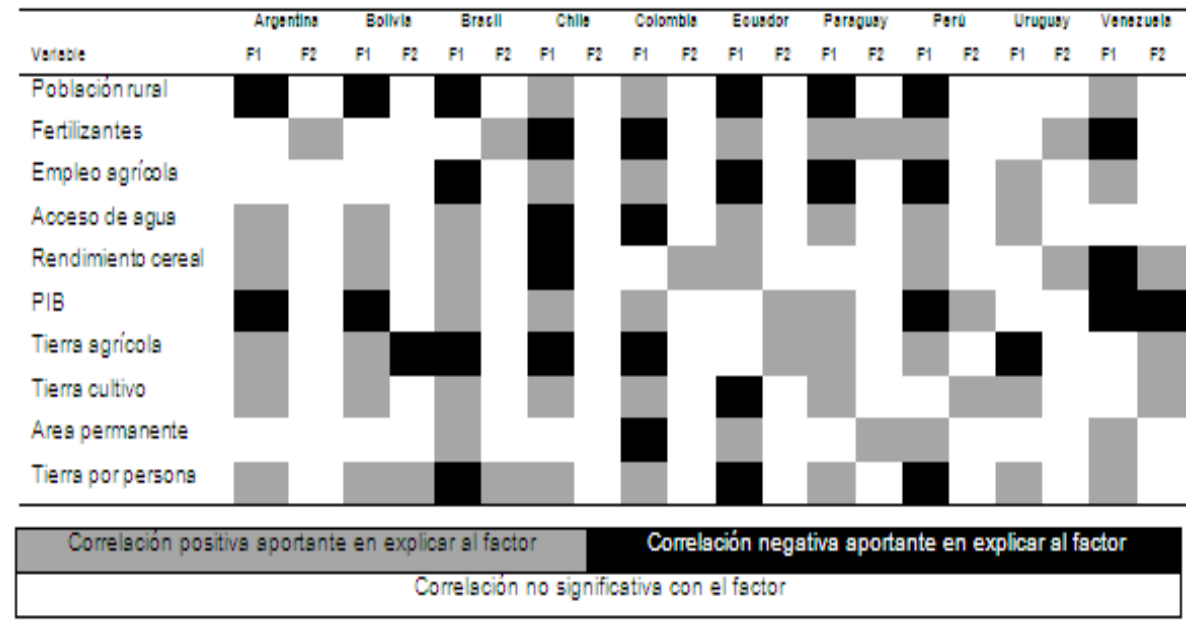

Fuente: los autores, 2014

De acuerdo con los resultados del análisis canónico, Chile y Uruguay se destacan como los países con un mejor desarrollo agrícola en las variables: suministro de agua, uso de fertilizantes, crecimiento del PIB agrícola y rendimiento de cereales. Venezuela, por su parte, es el país con el desempeño más modesto en estas variables.

El análisis canónico muestra, también, a Paraguay como el país con mejores indicadores en población rural y empleo rural. Uruguay y Argentina, por su parte, presentan el desempeño más pobre en estos aspectos. En cuanto al uso de la tierra agrícola, Bolivia se destaca como el país con los mayores avances en este indicador; mientras que Argentina, Uruguay, Perú y Chile son los de menor desempeño en este indicador.

Aunque el análisis de componentes principales determinó que no hay homogeneidad en las variables que se relacionan positivamente con el desarrollo agrícola en todos los países de la región, las variables que presentan una mayor relación positiva con éste en la mayoría de países son tierra cultivable como factor principal en siete países y como factor secundario en otros dos; tierra por persona como factor principal en siete países y como factor secundario en uno más; y acceso al agua como factor principal en siete países. 
Paraguay es el país con el mayor número de variables con relación positiva con su desarrollo agrícola en el factor principal. Uruguay, por su parte, es el país con el menor número de variables con relación negativa con el desarrollo agrícola en su factor principal; siendo a su vez el país con mayor cantidad de tierra cultivable. Chile, es el país que más se acerca en asociar todas las variables en único factor y de ahí su eficiencia; siendo a su vez el único país que presenta un decrecimiento significativo de la tierra cultivable.

\section{DISCUSIÓN}

El análisis de la dinámica del desarrollo agrícola en Suramérica presentó como resultado, en términos generales, la inexistencia de un patrón homogéneo de desarrollo en esta región. Se encontró que las variables con una mayor relación positiva con el desarrollo agrícola en la mayoría de países de Suramérica son: la tierra destinada a cultivos, la tierra por persona y el acceso al agua. Estos resultados concuerdan con los reportes de otros autores sobre indicadores relacionados con el desarrollo agrícola: uso del suelo (Lowe et al., 1993); tenencia de la tierra, gestión del agua en la agricultura (FAO, 2004).

Otro de los hallazgos de este estudio, está relacionado con las brechas existentes entre los países, en términos del desarrollo agrícola. Argentina y Paraguay se destacan como los países en los cuales se evidencia un crecimiento significativo en el mayor número de indicadores; ambos países presentan una mayor participación del sector agropecuario en el PIB y el rendimiento del cultivo de cereales frente al resto de países.

Según Kjöllerström (2004), estas brechas generadas en el desarrollo agrícola entre países tienen relación, entre otras cosas, con la misma heterogeneidad por ejemplo en el gasto público en las zonas rurales, como políticas examinadas más bien a nivel micro que desde una perspectiva macroeconómica.

Sin embargo, existen una serie de factores externos que pueden afectar seriamente el desempeño agrícola, visto desde una perspectiva macroeconómica, donde se destacan condiciones climáticas como el fenómeno de El Niño, conflictos entre países, precios internacionales y otras políticas públicas.

Algunos países han dado prioridad en sus territorios a la inversión en infraestructura, como Brasil y Perú, mientras que otros han asignado una mayor parte de los recursos a los servicios sociales tales como Argentina, mientras que en países como Bolivia, un $75 \%$ del gasto en el sector agrícola y áreas rurales, ha sido financiado por agencias internacionales, disminuyendo el rol del Estado en este sector (Kjöllerström, 2004).

Adicionalmente, estas brechas están relacionadas con la atención que se brinda al sector agrícola en cada país, por cuanto en algunos casos, los Estados privilegian el abastecimiento a bajos precios sobre el desarrollo agrícola interno, lo cual para casos como el de Venezuela, de acuerdo con Hernández (2009), representa además un sistema con altos subsidios a la red de distribución pública, en el cual hay un claro beneficio para los consumidores de bajos ingresos, en perjuicio del desarrollo agrícola interno y la soberanía alimentaria.

Un aspecto que puede explicar el buen desempeño general en el desarrollo agrícola de Paraguay a partir del estudio de las variables propuestas, es el hecho presentar el mejor indicador en uso del suelo agrícola, por cuanto de acuerdo con 
los hallazgos de Rodríguez (2011), un factor que incide en el pobre desempeño agrícola de los países y en la generación de empleo, es la tenencia de la tierra; a lo cual, Deininger (2004) agrega que es necesario mejorar el funcionamiento de los mercados de tierras, al igual que los seguros y créditos para incrementar los bajos ingresos de los trabajadores.

La evolución en el desarrollo del sector agrícola argentino, entre diversas razones, obedeció a su plan de conversión económica implementado al comienzo de la década del noventa. La implementación de medidas macroeconómicas basadas en políticas crediticia y monetaria, la reducción de aranceles no solo a los alimentos, sino también a insumos y maquinaria, una política de empleo, pero sobre todo de integración regional, sin duda favorecieron el crecimiento del sector agrícola (Ghezán, Mateos y Everdín, 2001).

Adicionalmente y junto con Brasil, Argentina cuenta con uno de los mejores sistemas de investigación agrícola y transferencia de tecnología en América Latina (Kjöllerström, 2004), lo cual le ha permitido la incorporación de tecnología y la transformación substancial de la producción agrícola (Ghezán, Mateos y Everdín, 2001).

Por su parte, el buen desempeño general de Argentina en el desarrollo agrícola puede estar relacionado con presentar el mejor indicador en generación de empleo rural, pues de acuerdo con los hallazgos de Barrientos y Castrillón (2007), la generación de empleos agrícolas está relacionada con el incremento de las unidades agrarias productivas y agroindustriales, aspectos relacionados a su vez con el acceso a las tierras mencionado anteriormente.

Sin embargo y de acuerdo con lo planteado por Michelini (2013), las políticas públicas orientadas a la promoción de la agroindustria de gran escala, al margen de los buenos resultados en las variables del desarrollo agrícola, están incrementando la marginalización de los pequeños productores y no están contribuyendo a la reducción de la pobreza en el área rural. Es allí, entonces, donde debe existir un equilibrio entre la agricultura campesina y empresarial, pero también en los mecanismos de acceso a los mercados por parte de la producción campesina, siempre que cuenten con propiedad de la tierra, logrando acceder a otro tipo de recursos y beneficios de organismos públicos y privados, buscando mejorar la productividad, pero también las condiciones de empleo rural, la rentabilidad de las unidades de producción, la calidad de vida (Cazalis, 1980), entre otras variables necesarias para el desarrollo agrario.

De acuerdo con los resultados del análisis canónico, Chile y Uruguay se destacan como los países con un mejor desarrollo agrícola en las variables: suministro de agua, uso de fertilizantes, crecimiento del PIB agrícola y rendimiento de cereales. Para el caso de Chile y de acuerdo con los hallazgos de Pisani y Franceschetti (2010), las políticas públicas orientadas a la agricultura de exportación, el desarrollo de clústers, la planificación del desarrollo con enfoque territorial, el capital institucional, la formación del capital humano y la globalización de la economía, han permitido importantes progresos en el sector agrícola.

Para el caso de Uruguay y de acuerdo con los reportes del MGAP (2011), estos resultados se deben a las diferentes políticas públicas establecidas por el gobierno, como asistencia técnica, extensión rural, cadenas de generación de valor, creación de la dirección nacional de desarrollo rural y la ley de descentralización del Ministerio, que han permitido lograr una reducción de la pobreza y de las necesidades básicas insatisfechas, de manera sostenida, particularmente en la primera década del siglo XXI. 
Paraguay, de acuerdo con el análisis canónico, se ubicó como el país con mejores indicadores en población rural y empleo rural; no obstante y de acuerdo con los reportes de la FIAN (2006), en este país se presenta un contraste entre un sector agrícola próspero orientado a la exportación y una reducción del nivel de vida de la agricultura campesina, con un incremento de campesinos en extrema pobreza.

El análisis canónico arrojó, también, que Bolivia es el país con un mejor indicador en cuanto al uso de la tierra agrícola. Sin embargo, de acuerdo con los reportes de Arias y Salas (2006), en este país se ha presentado un fenómeno de reducción en el tamaño de la propiedad agrícola, el cual acompañado de un decremento en la productividad como consecuencia del manejo inadecuado e intensivo del suelo y el agua, han llevado al sector agrícola a una situación de pobreza estructural, con niveles de incidencia de la pobreza rural que alcanzan al 93\% de los hogares. En este sentido, Rigg (2006) concluye que las reformas agrarias orientadas solo a la redistribución de la tierra no ofrecen una solución a la reducción de la pobreza rural.

Este último tema, se enmarca también en la actualidad colombiana, en la cual en la implementación de la Ley de Tierras, de restitución y de titulación de baldíos, entre otros, según las experiencias de Paraguay y Bolivia, no sería suficiente garantía para apalancar procesos de desarrollo, considerando el conglomerado de variables que efectivamente pueden tener incidencia, sobre la base de una visión multidimensional de los territorios rurales.

Aunque el análisis de componentes principales determinó que no hay homogeneidad en las variables que se relacionan positivamente con el desarrollo agrícola en todos los países de la región, las variables que presentan una mayor relación positiva con éste en la mayoría de países son: tierra cultivo como factor principal en siete países y como factor secundario en otros dos; tierra por persona como factor principal en siete países y como factor secundario en uno más; y acceso al agua como factor principal en siete países.

Estos resultados aportan al entendimiento de la dinámica del desarrollo y pueden orientar la implementación de políticas que apunten a un desarrollo agrícola sostenible, pues de acuerdo con los hallazgos de Pachón y Argüello (2010), éste es uno de los principales factores percibidos por los productores como fundamentales para el desarrollo. Estas políticas de desarrollo agrícola sin embargo, no deben implementarse de manera generalizada sino localizada, para detener la degradación de los servicios naturales asociados al uso de la tierra y el agua en la agricultura, en aquellas regiones con alto potencial (Carreño, Frank y Viglizzoa, 2012).

Estas variables por otro lado, corroboran las proyecciones de la CEPAL, FAO e IICA (2011), que auguran una tendencia al crecimiento de la agricultura en Suramérica, orientada por el incremento en la demanda mundial de alimentos y basada en la disponibilidad de tierra de la región que puede ser incorporada a la agricultura con un recurso hídrico abundante. Este crecimiento de la agricultura sin embargo, depende del cierre de brechas tecnológicas para incrementar el rendimiento de los cultivos y aumentar la producción de alimentos.

Un factor que no se consideró en el análisis y que debe incluirse en futuros estudios es el capital social, por cuanto de acuerdo con los hallazgos de Esparcia (2014), más allá del soporte externo por parte del Estado o las organizaciones no gubernamentales, se debe apuntar a la sostenibilidad de los procesos de desarrollo agrícola y la consolidación de redes de actores del desarrollo; de igual forma, como lo plantean Fawaz y Vallejos (2011), el fortalecimiento del capital social es un factor que contribuye al éxito de los programas de desarrollo agrícola; 
y de acuerdo con los hallazgos de McDonald et al. (2013), el desarrollo de capacidades locales contribuye al desarrollo de la acción colectiva y capital político para mejorar la respuesta integrada al declive de las condiciones de vida en el campo.

\section{CONCLUSIONES}

La evaluación de los indicadores del desarrollo de la agricultura en Suramérica, permitió establecer que las variables que presentan una mayor relación positiva con el desarrollo agrícola en la mayoría de países de Suramérica son la tierra destinada a cultivos, la tierra por persona y el acceso al agua, resultados que aportan al entendimiento de la dinámica del desarrollo y pueden orientar la implementación de políticas que apunten a un desarrollo agrícola sostenible, teniendo en cuenta que Suramérica cuenta con gran disponibilidad de tierra que puede ser incorporada a la agricultura y cuenta además con un recurso hídrico abundante. Adicionalmente, se encontró que no existe un patrón de desarrollo de la agricultura en esta región, en la cual Argentina y Paraguay presentan un crecimiento significativo en el mayor número de indicadores analizados; siendo estos países los que presentan una mayor participación del sector agropecuario en el PIB.

\section{CONFLICTO DE INTERESES}

Los autores manifiestan que no existe ningún conflicto de intereses para la publicación de este artículo.

\section{FUENTES DE FINANCIACIÓN}

Esta investigación fue financiada por la Universidad de Antioquia.

\section{REFERENCIAS}

- Arias, J. y Salas, V. (2006). Las experiencias de negociación agrícola entre la Comunidad Andina de Naciones (CAN) y la Unión Europea (UE). Revista Intercambio, VIII.

- Armstrong, H. y Taylor, J. (2000). Regional economics and policy. Oxford: Blackwell, Publisher.

- Barrientos, J. y Castrillón, G. (2007). Generación de empleo en el sector agrario colombiano. Agronomía Colombiana, 25(2), 383-395.

- Baudasse, T. y Calderón, C. (2009). Integración comercial del sector agrícola y desigualdad económica en los países en vías de desarrollo. Inv. Econ., 68(269), 37-72.

- Carreño, L., Frank, F. y Viglizzoa, E. (2012). Tradeoffs between economic and ecosystem services in Argentina during 50 years of land-use change. Agriculture, Ecosystems and Environment, 154, 68-77. 
- Cazalis, M. (1980). Agricultural development. Revista de Estudios AgroSociales, 112, 21-37.

- CEPAL, FAO e IICA (2011). Perspectivas de la agricultura y del desarrollo rural en las Américas: una mirada hacia América Latina y el Caribe 20112012. San José, C.R.: IICA.

- Costa, C., Almeida, A., Ferreira, M. y Silva, E. (2013). Determinantes do desenvolvimento do setor agropecuário nos municípios. Rev. Adm. (São Paulo), 48(2), 295-309.

- Deininger, K. (2004). Land Policies and Land Reform. Washington: World Bank.

- Delgadillo, N. y Chalita, L. (2013). La política agrícola en México durante el periodo 1995-2009: un análisis multivariado. Rev. Mex. Cienc. Agríc., 4(2), 307-313.

- Esparcia, J. (2014). Innovation and networks in rural areas. An analysis from European innovative projects. Journal of Rural Studies, 34, 1-14.

- FAO. (2004). Política de desarrollo agrícola: conceptos y principios. Roma: FAO.

- Fawaz, J. y Vallejos, R. (2011). Calidad de vida, ocupación, participación y roles de género: un sistema de indicadores sociales de sostenibilidad rural (Chile). Cuad. Desarro. Rural, 8(67), 45-68.

- FIAN. (2006). La reforma agraria en Paraguay. Informe de la misión investigadora sobre el estado de la realización de la reforma agraria en tanto obligación de derechos humanos. Germany: FIAN International. Recuperado de http://www2.ohchr.org/english/bodies/cescr/docs/infongos/fianparaguay.pdf

- Ghezán, G., Mateos, M. y Everdín, J. (2001). Impact of policy of structural adjusting in agricultural and agroindustrial sectors: The Argentina Case. Serie Desarrollo Productivo, No. 90. Santiago, Chile: Economic Commission for Latin America and the Caribbean -ECLAC-.

- Gorenstein, S., Napal, M. y Olea, M. (2007). Territorios agrarios y realidades rururbanas. Reflexiones sobre el desarrollo rural a partir del caso pampeano bonaerense. Revista Eure, 33(100), 91-113.

- Hernández, J. (2009). Evolución y resultados del sector agroalimentario en la V República. CDC, 26(72), 67-100.

- Kjöllerström, M. (2004).Competitividad del sector agrícola y pobreza rural: el papel de los gastos públicos en América Latina. Series Desarrollo Productivo, No. 155. Santiago, Chile: Economic Commission for Latin America and the Caribbean -ECLAC-.

- Lowe, P., Murdoch, J., Marsden, T., Munton, R. y Flynn, A. (1993). Regulating the new rural spaces: the uneven development of land. Journal of Rural Studies, 9(3), 205-222.

- Ministerio de Ganadería, Agricultura y Pesca -MGAP-. (2011). Proyecto Uruguay Rural, aportes a la construcción del desarrollo rural: síntesis de resultados y lecciones aprendidas período 2005-2011. Uruguay: Dirección General de Desarrollo Rural -DGDR-. Recuperado de http://www.mgap.gub.uy/portal/agxppdwn.aspx?

- 7,10,262,O,S,0,4875\%3BS\%3B1\%3B156

- Michelini, J. (2013). Small farmers and social capital in development projects: Lessons from failures in Argentina's rural periphery. Journal of Rural Studies, 30, 99-109.

- McDonald, C., Kirk-Brown, A., Frost, L., Van Dijk, P. y Rainnie, A. (2013). Partnerships and integrated responses to rural decline: The role of collective efficacy and political capital in Northwest Tasmania, Australia. Journal of Rural Studies, 32, 346-356. 
- Moulaert, F., Sekia, F. y Boyabe, J. (1999). Innovative region, social region? An alternative view of regional innovation. En European Meeting on Applied Evolutionary Economics. France: Grenoble.

- Norton, R. (1992). Integration of food and agricultural policy with macroeconomic policy: methodological considerations in a Latin American perspective. Roma: FAO.

- Pachón, F. y Argüello, M. (2010). Rural development perceptions between farmers and policy executors in the Tequendama Province (Cundinamarca, Colombia). Agron. Colomb., 28(3), 524-530.

- Pisani, E. y Franceschetti, G. (2010). Da agrariedade á ruralidade: Novos enfoques á economia territorial. Economía, XXXV(30), 79-101.

- Porter, M. (2007). La ventaja competitiva de las naciones. Harvard Business Review, 4-23. Recuperado de http://es.slideshare.net/ivanss3/laventaja-competitiva-de-las-naciones-michael-e-porter

- Rigg, J. (2006). Land, Farming, Livelihoods, and Poverty: Rethinking the Links in the Rural South. World Development, 34(1), 180-202.

- Rodríguez, J. (2011). Vías de desarrollo, cambio tecnológico y políticas estructurales en la agricultura moderna venezolana. CDC, 28(76), 67-96.

- Soto, C. (2003). La agricultura comercial de los distritos de riego en México y su impacto en el desarrollo agrícola. Invest. Geog., 50, 173-195.

- Terluin, I. (2003). Differences in economic development in rural regions of advanced countries: an overview and critical analysis of theories. Journal of Rural Studies, 19, 327-344.

1. Ing. Agric., MSc, PhD, Docente, Grupo GaMMA, Facultad de Ciencias Agrarias, Universidad de Antioquia. Medellín, Colombia. holmes.rodriguez@udea.edu.co

2. I. A., MSc., Docente, Grupo GISER, Facultad de Ciencias Agrarias, Universidad de Antioquia. Medellín, Colombia. cajramirezgo@unal.edu.co

3. Estad., Esp., Docente, Grupo GISER, Facultad de Ciencias Agrarias, Universidad de Antioquia. Medellín, Colombia. frbstatistical@yahoo.es

Para citar este artículo: Rodríguez Espinosa, H., Ramírez Gómez, C. J. y Restrepo-Betancur, L. F. (2016). Análisis comparativo de la dinámica de desarrollo agrícola en Suramérica en el período 1980-2010. Revista Luna Azul, 42, 15-29. Recuperado http://http://200.21.104.25/lunazul/index.php?option=com_content\&view=article\&id $=125$ 